\title{
Mach-Zehnder Based on Large Knot Fiber Resonator for Refractive Index Measurement
}

\author{
André D. Gomes and Orlando Frazão
}

\begin{abstract}
A Mach-Zehnder sensor based on a large knot fiber resonator with a diameter of a few millimeters is designed using a single long taper. The long taper of some centimeters is fabricated with a $\mathrm{CO}_{2}$ laser technique. In air, light cannot couple between adjacent sections in the knot, and no signal is observed. However, in liquid, light is less confined and there is coupling between adjacent sections of the knot, resulting in a phase difference and consequent interference. The Mach-Zehnder is formed by the two contact points in the knot. The refractive index sensing of liquid compounds is achieved by monitoring the wavelength shift of the spectra. A sensitivity of $642 \pm 29 \mathrm{~nm} /$ refractive index unit (RIU) is achieved for refractive index sensing in the range of 1.3735-1.428 with a resolution of 0.009 RIU. For temperature sensing, a sensitivity of $-42 \pm 9 \mathrm{pm} /{ }^{\circ} \mathrm{C}$ is observed. A low influence of temperature in the refractive index change is observed: $6.5 \times 10^{-5} \mathrm{RIU}^{\circ} \mathrm{C}$.
\end{abstract}

Index Terms-Large knot fiber resonator, Mach-Zehnder, optical fiber sensor, refractive index sensor.

\section{INTRODUCTION}

$\mathbf{R}$ ECENTLY, optical refractive index sensors or refractometers based on all-fiber interferometers [1] or resonators [2] have been studied and developed due to their high sensitivity, broad measurement range and compact size [2]. From these all-fiber based interferometric sensors, the all-fiber Mach-Zehnder interferometers (MZI) have received considerable attention [1], [4]-[7].

The MZI technique is used for measuring the refractive index of a medium due to the induced phase shift of the guided light created by the analyzed medium [3].

For this purpose, Tian et al. [1] presented a single-mode fiber (SMF)-based MZI by simply concatenating two fiber tapers separated by a short $(24-55 \mathrm{~mm})$ middle section, obtaining $17.1 \mathrm{~nm} / \mathrm{RIU}$ (RIU - Refractive Index Unit) of sensitivity. Lu et al. [4] used a tapered fiber MZI created on single-mode fiber by simple fusion splicing for the simultaneous measurement of refractive index and temperature. They achieved sensitivities of $-23.188 \mathrm{~nm} / \mathrm{RIU}$ and $0.071 \mathrm{~nm} /{ }^{\circ} \mathrm{C}$ for refractive index and temperature sensing, respectively. In 2012, an all-fiber in-line single mode-multimode-thinned single

Manuscript received January 18, 2016; revised February 22, 2016; accepted March 3, 2016. Date of publication March 7, 2016; date of current version April 20, 2016.

The authors are with the Instituto de Engenharia de Sistemas e Computadores, Tecnologia e Ciência, Porto 4169-007, Portugal, and also with the Department of Physics and Astronomy, Faculty of Sciences, University of Porto, Porto 4169-007, Portugal (e-mail: ardcgomes@gmail.com; ofrazao@inescporto.pt).

Color versions of one or more of the figures in this letter are available online at http://ieeexplore.iee.org.

Digital Object Identifier 10.1109/LPT.2016.2538963 mode (SM-MM-TSM) MZI fiber structure was developed, using only a splicing method, obtaining a sensitivity of $-25.2935 \mathrm{~nm} / \mathrm{RIU}$ for refractive index and $61.5 \mathrm{pm} /{ }^{\circ} \mathrm{C}$ for temperature sensing [7].

Other fiber sensors have been developed with this purpose. For example, a biconical fiber taper sensor proposed by Kieu and Mansuripur [8], which presents sensitivities of $\sim 650 \mathrm{~nm} / \mathrm{RIU}$ and $\sim 10 \mathrm{pm} /{ }^{\circ} \mathrm{C}$, was applied for refractive index and temperature sensing. Moreover, a MZI embedded in a taper was reported with a refractive index sensitivity of $2210.84 \mathrm{~nm} / \mathrm{RIU}$, good for low index variation sensing, and temperature sensitivity of $-6.66 \mathrm{pm} /{ }^{\circ} \mathrm{C}$ [14].

There has also been a great interest in the past few years in microfiber resonators applied as sensing elements. To this class of devices belong the knot resonators, which are made tying a knot in an optical microfiber taper, creating a ring geometry with micrometer dimensions [9]. The taper in the knotted zone allows the evanescent field of light to couple between the adjacent sections of the taper creating a resonance [10]. This overlap of the fiber with itself does not require a precise alignment [9].

In the temperature sensing field, a comparison between a silica microfiber knot resonator (MKR) and a polymer MKR is presented by Wu et al. [11], reaching a sensitivity of $52 \mathrm{pm} /{ }^{\circ} \mathrm{C}$ with a $190 \mu$ m-diameter silica MKR, assembled using a $1.7 \mu$ m-diameter microfiber; and a sensitivity of $-266 \mathrm{pm} /{ }^{\circ} \mathrm{C}$ with a $98 \mu \mathrm{m}$-diameter polymer MKR, assembled using a $2.1 \mu \mathrm{m}$ polymer microfiber. For refractive index sensing, Lim et al. [12] proposed a MKR with a diameter of $\sim 0.5 \mathrm{~mm}$ in a Sagnac loop reflector, obtaining a RI sensitivity of $30.49 \mathrm{~nm} / \mathrm{RIU}$ and a temperature sensitivity of $20.6 \mathrm{pm} /{ }^{\circ} \mathrm{C}$. Yu et al. [13] reported a polymer MKR for refractive index sensing achieving a sensitivity of about $95.5 \mathrm{~nm} / \mathrm{RIU}$. In the last year, a cascaded MKR with a sensitivity of $6523 \mathrm{~nm} / \mathrm{RIU}$ was presented, suitable for slight refractive index variations [15].

In this letter, a Mach-Zehnder based on a large knot fiber resonator (MZ-LKR) with a diameter of a few millimeters is presented for refractive index sensing of liquids. The knot is made on a taper with around $60 \mu \mathrm{m}$-diameter fabricated with a $\mathrm{CO}_{2}$ laser.

\section{Experimental Setup AND Results}

Figure 1a) shows the experimental setup. It consists in a broadband source with a central wavelength of $1550 \mathrm{~nm}$ and a bandwidth of $100 \mathrm{~nm}$ connected to an optical fiber where the MK-LKR is located. The transmitted signal spectrum is 
a)

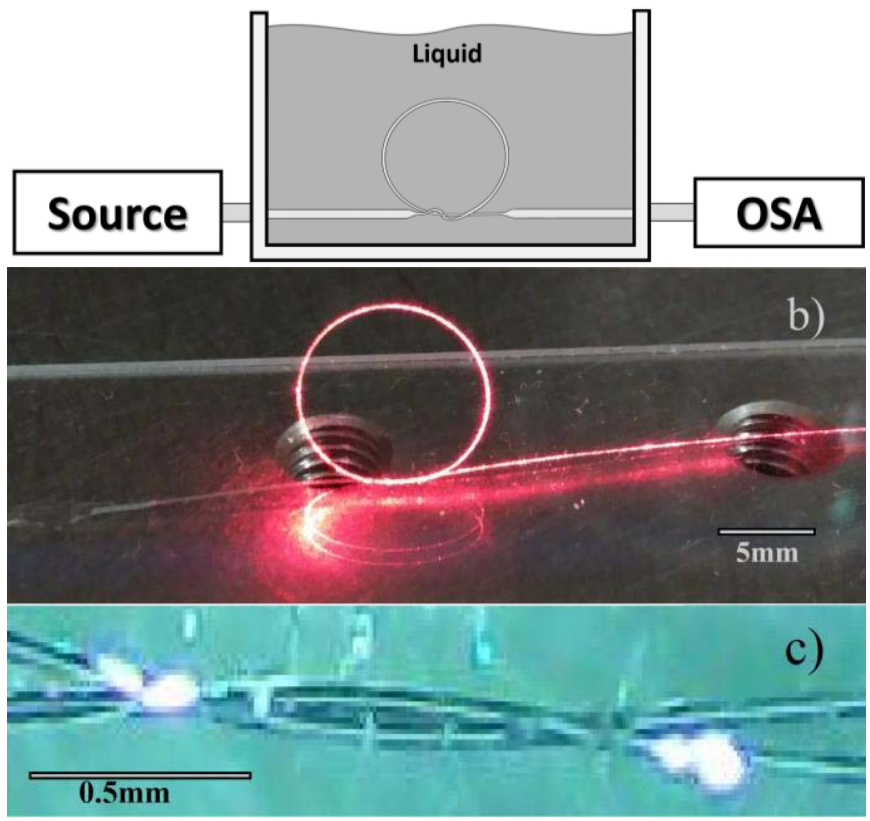

Fig. 1. a) Experimental setup. b) Mach-Zehnder large knot resonator in air, illuminated by a He-Ne laser. c) Photograph of a knot of a Mach-Zehnder large knot resonator with $3.5 \mathrm{~mm}$ diameter.

observed in an optical spectrum analyzer (OSA) with a $1 \mathrm{~nm}$ resolution. The sensor is immersed in liquid. This allows it to be studied under different liquids with different refractive indices. The setup also allows a gradual heating of the liquid. The sensor is made manually by tying a knot in a tapered single mode optical fiber (SMF28). The taper was fabricated using a $\mathrm{CO}_{2}$ laser to reduce the fiber diameter to $\sim 60 \mu \mathrm{m}$. The diameter of the ring is reduced until it reaches a value of $\sim 3.5 \mathrm{~mm}$. The knot is located near the transition zone between the untapered SMF and the tapered section.

In Figure 1b) a $1 \mathrm{~cm}$-diameter MZ-LKR illuminated by a $\mathrm{He}-\mathrm{Ne}$ laser is shown.

The transmitted signal spectrum of a $3.5 \mathrm{~mm}$ diameter MZ-LKR, in air and in liquid (ethylene glycol, $n=1.426$ ), illuminated by a broadband source can be observed in Figure 2 . The MZ-LKR interferometer is formed by the two contact points in the knot, visible in Figure 1c).

The mode of operation of the MZ-LKR is the following: in air, light travels in the ring and is lost in the loop and in the knot due to curvature losses. Around the two contact points in the knot, the presence of air does not allow light to be coupled between adjacent sections because of the high confinement of light due to large taper diameter dimensions. Hence, the light cannot pass directly to the output of the ring and a low power signal is observed. When the sensor is immersed in liquid, light in the contact points of the knot is now less confined and capable of coupling between adjacent fibers, with a certain phase difference, giving rise to an interference spectrum. This interference is visible in Figure 2. Part of the light is now capable of passing directly to the output of the ring, without losing itself in the loop, and a spectral signal with higher intensity is observed in the OSA.

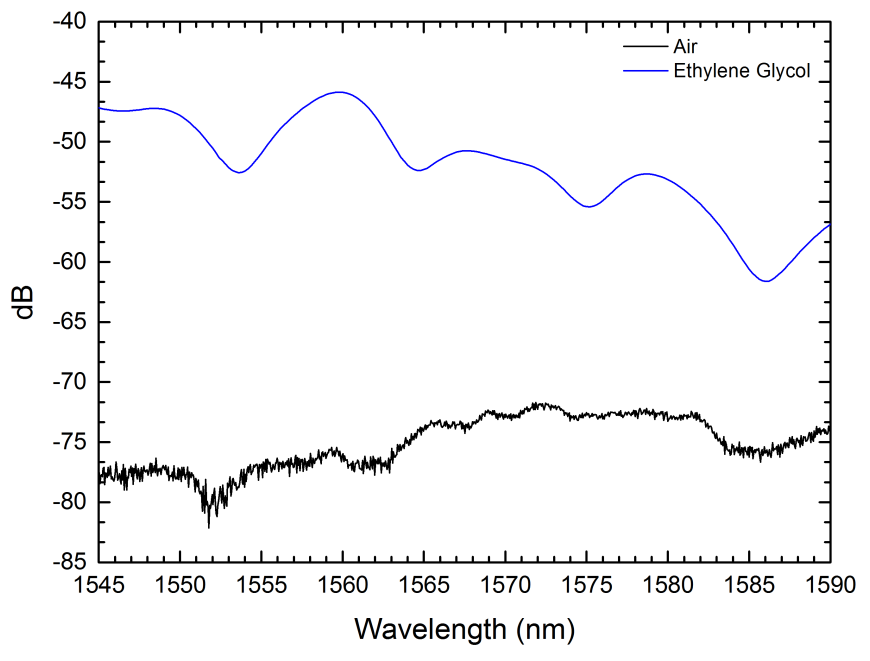

Fig. 2. Transmitted signal of the MZ-LKR when it is in air and when it is immersed in ethylene glycol $(n=1.428)$. The free spectral range (FSR) in ethylene glycol is approximately $10.15 \mathrm{~nm}$.

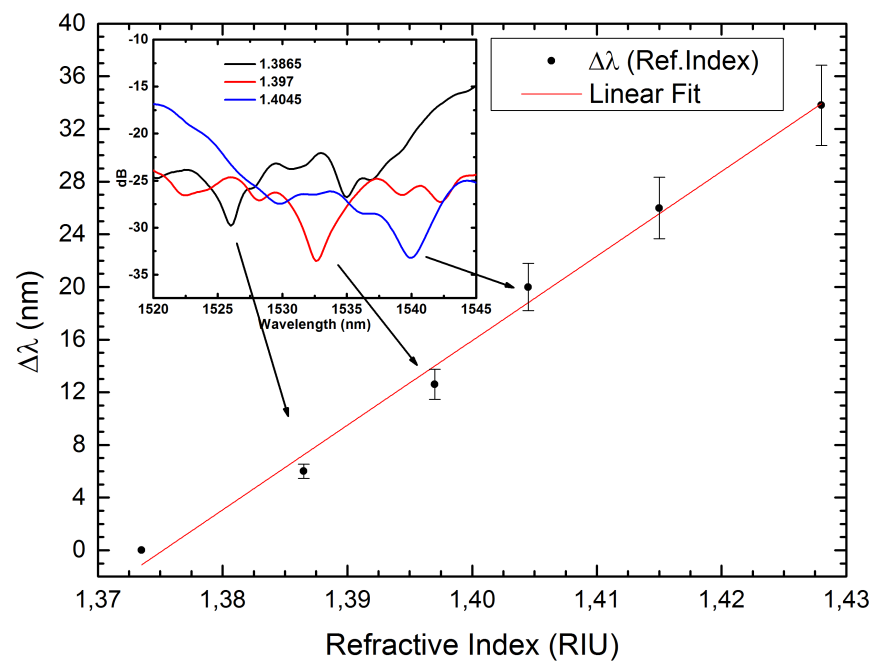

Fig. 3. Wavelength shift as function of refractive index. The inner plot shows the spectra of the transmitted signal for three different refractive index.

The peak shift of the transmitted signal spectrum from the sensor was studied by dipping the sensor in ethylene glycol mixtures with different refractive indices [16]. In Figure 3 the wavelength shift as function of the refractive index is presented. As the wavelength shift is directly proportional to the refractive index [3], a linear fit was applied. From this fit, a sensitivity of $642 \pm 29 \mathrm{~nm} / \mathrm{RIU}$ was obtained. The auxiliary graph in Figure 3 clearly shows the wavelength shift due to refractive index change.

The study of the wavelength shift as a function of temperature variations was also performed. The MZ-LKR was immersed in water whose temperature was progressively increased. The wavelength shift as a function of temperature is depicted in Figure 4. A linear fit was performed on the data. From this fit, a sensitivity of $-42 \pm 9 \mathrm{pm} /{ }^{\circ} \mathrm{C}$ was obtained. The negative sensitivity is related to the knot dimension, being the thermo-optical effect dominant in this case. Dividing both sensitivities, a value of $6.5 \times 10^{-5} \mathrm{RIU} /{ }^{\circ} \mathrm{C}$ is reached, 


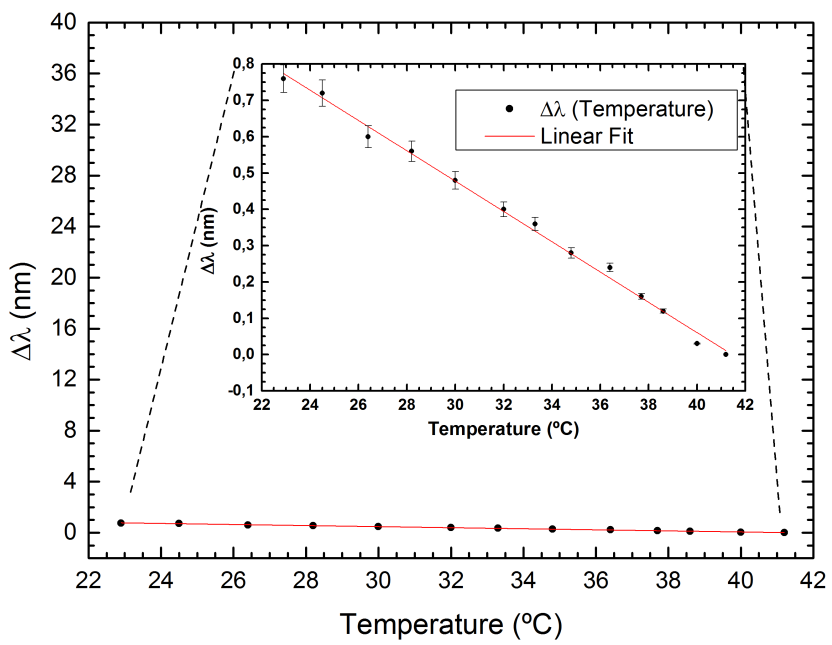

Fig. 4. Wavelength shift as a function of temperature.

TABLE I

COMPARISON BETWEen DifFERENT CONFIGURATIONS

\begin{tabular}{lll}
\hline \hline \multirow{2}{*}{ Author } & \multicolumn{1}{c}{ Type } & \multicolumn{1}{c}{ Sensitivity } \\
\hline This work & Large Knot Resonator & $\mathbf{6 4 2 n m} /$ RIU \\
{$[12]$} & MKR in Sagnac loop & $30.49 \mathrm{~nm} /$ RIU \\
{$[13]$} & Polymer MKR & $95.5 \mathrm{~nm} / \mathrm{RIU}$ \\
{$[4]$} & Tapered Fiber MZI & $-23.188 \mathrm{~nm} / \mathrm{RIU}$ \\
{$[7]$} & SM-MM-TSM MZI & $-25.2935 \mathrm{~nm} / \mathrm{RIU}$ \\
{$[1]$} & SMF based MZI & $17.1 \mathrm{~nm} / \mathrm{RIU}$ \\
{$[8]$} & Biconical fiber taper & $\sim 650 \mathrm{~nm} / \mathrm{RIU}$ \\
{$[14]$} & MZI embedded in a taper & $2210.84 \mathrm{~nm} / \mathrm{RIU}$ \\
{$[15]$} & Cascaded MKR & $6523 \mathrm{~nm} / \mathrm{RIU}$ \\
& & \\
This work & Large Knot Resonator & $\mathbf{- 4 2 p m} /{ }^{\circ} \mathrm{C}$ \\
{$[12]$} & MKR in Sagnac loop & $20.6 \mathrm{pm} /{ }^{\circ} \mathrm{C}$ \\
{$[11]$} & Simple polymer MKR & $-266 \mathrm{pm} /{ }^{\circ} \mathrm{C}$ \\
{$[4]$} & Tapered Fiber MZI & $71 \mathrm{pm} /{ }^{\circ} \mathrm{C}$ \\
{$[7]$} & SM-MM-TSM MZI & $61.5 \mathrm{pm} /{ }^{\circ} \mathrm{C}$ \\
{$[8]$} & Biconical fiber taper & $\sim 10 \mathrm{pm} /{ }^{\circ} \mathrm{C}$ \\
{$[14]$} & MZI embedded in a taper & $-6.66 \mathrm{pm} /{ }^{\circ} \mathrm{C}$ \\
& & \\
\hline \hline
\end{tabular}

showing a low influence of temperature in the refractive index measurement.

\section{CONCLUSION}

The refractive index sensing of liquids was achieved with a MZ-LKR. For refractive index sensing, a sensitivity of $642 \pm 29 \mathrm{~nm} / \mathrm{RIU}$ was obtained in a refractive index range from 1.3735 to 1.428 with a resolution of 0.009 RIU. Comparing the sensitivity with previous published results and with other different configurations, as shown in Table 1, the MZ-LKR stands in a zone where most of the remaining configurations have less sensitivity, except the MZI embedded in a taper [14] and the cascaded MZI configuration [15]. This sensor presents a similar sensitivity to the biconical fiber taper [8].
For temperature sensing, a sensitivity of $-42 \mathrm{pm} /{ }^{\circ} \mathrm{C}$ was observed. This sensitivity is of the same order as the already published MKR and MZI, except for the polymer MKR [11] which reveals a significant increase in the sensitivity due to the polymer. The size of the MZ-LKR allows it to be easily made and manageable. The sensor is more resistant due to its dimensions, revealing an advantage in relation to the micrometer size knot resonators. On the other hand, the MZ-LKR shows a higher sensitivity to refractive index variations than most of the other MKR and MZI.

Further studies should be performed to investigate the influence of the number of contact points in the knot in the signal spectrum, as well as to determine if there is any predominance of one contact point over the other in the interference spectrum.

\section{REFERENCES}

[1] Z. Tian et al., "Refractive index sensing with Mach-Zehnder interferometer based on concatenating two single-mode fiber tapers," IEEE Photon. Technol. Lett., vol. 20, no. 8, pp. 626-628, Apr. 15, 2008.

[2] X. Li and H. Ding, "A stable evanescent field-based microfiber knot resonator refractive index sensor," IEEE Photon. Technol. Lett., vol. 26 , no. 16, pp. 1625-1628, Aug. 15, 2014.

[3] T. Schubert, N. Haase, H. Kück, and R. Gottfried-Gottfried, "Refractiveindex measurements using an integrated Mach-Zehnder interferometer," Sens. Actuators A, Phys., vol. 60, nos. 1-3, pp. 108-112, 1997.

[4] P. Lu, L. Men, K. Sooley, and Q. Chen, "Tapered fiber Mach-Zehnder interferometer for simultaneous measurement of refractive index and temperature," Appl. Phys. Lett., vol. 94, no. 13, p. 131110, 2009.

[5] H. Y. Choi, M. J. Kim, and B. H. Lee, "All-fiber Mach-Zehnder type interferometers formed in photonic crystal fiber," Opt. Exp., vol. 15, no. 9 , pp. 5711-5720, 2007.

[6] J. H. Lim, H. S. Jang, K. S. Lee, J. C. Kim, and B. H. Lee, "MachZehnder interferometer formed in a photonic crystal fiber based on a pair of long-period fiber gratings," Opt. Lett., vol. 29, no. 4, pp. 346-348, 2004.

[7] L. Li, L. Xia, Z. Xie, and D. Liu, "All-fiber Mach-Zehnder interferometers for sensing applications," Opt. Exp., vol. 20, no. 10, pp. 11109-11120, 2012.

[8] K. Q. Kieu and M. Mansuripur, "Biconical fiber taper sensors," IEEE Photon. Technol. Lett., vol. 18, no. 21, pp. 2239-2241, Nov. 1, 2006.

[9] L. Xiao and T. A. Birks, "High finesse microfiber knot resonators made from double-ended tapered fibers," Opt. Lett., vol. 36, no. 7, pp. 1098-1100, 2011.

[10] X. Jiang et al., "Demonstration of optical microfiber knot resonators," Appl. Phys. Lett., vol. 88, no. 22, p. 223501, 2006.

[11] Y. Wu, Y.-J. Rao, Y.-H. Chen, and Y. Gong, "Miniature fiber-optic temperature sensors based on silica/polymer microfiber knot resonators," Opt. Exp., vol. 17, no. 20, pp. 18142-18147, 2009.

[12] K.-S. Lim, I. Aryanfar, W.-Y. Chong, Y.-K. Cheong, S. W. Harun, and H. Ahmad, "Integrated microfibre device for refractive index and temperature sensing," Sensors, vol. 12, no. 9, pp. 11782-11789, 2012.

[13] H. Yu et al., "Solution concentration and refractive index sensing based on polymer microfiber knot resonator," Appl. Phys. Exp., vol. 7, no. 2, pp. 3-7, 2014.

[14] J. Yang et al., "High sensitivity of taper-based Mach-Zehnder interferometer embedded in a thinned optical fiber for refractive index sensing," Appl. Opt., vol. 50, no. 28, pp. 5503-5507, 2011.

[15] Z. Xu et al., "Highly sensitive refractive index sensor based on cascaded microfiber knots with Vernier effect," Opt. Exp., vol. 23, no. 5, pp. 6662-6672, 2015.

[16] O. Esteban, M. Cruz-Navarrete, A. González-Cano, and E. Bernabeu, "Measurement of the degree of salinity of water with a fiber-optic sensor," Appl. Opt., vol. 38, no. 25, pp. 5267-5271, 1999. 